\title{
Implementación de una estrategia de acceso abierto en la universidad: El caso de la UNVM (Argentina)
}

\author{
Mauricio Alejandro Grasso \\ Lila Isabel Pagola \\ Universidad Nacional de Villa María - UNVM, Argentina
}

\author{
Agustín Zanotti
}

Universidad Nacional de Córdoba - UNC, Argentina

CASE REPORT

\begin{abstract}
Resumen
Objetivo. El artículo aborda la experiencia de implementación de una estrategia de Acceso Abierto en la Universidad Nacional de Villa María, Argentina. Dicha iniciativa se enmarca en la Ley 26.899 "Creación de Repositorios Digitales Institucionales de Acceso Abierto, Propios o Compartidos" (2013), la cual establece la puesta en disponibilidad de toda producción científica financiada con fondos públicos.

Metodología. Basados en la propia participación como miembros del Programa: Producción y Circulación del Conocimiento en la Universidad Pública (PyCCUP), el trabajo se presenta como análisis de caso basado en una sistematización de experiencias.

Resultados. Los resultados detallan cuatro momentos involucrados en este proceso: 1. diagnóstico, 2. sensibilización, capacitación y conciliación de intereses 3. adecuación institucional, y 4. aspectos técnicos y de diseño.

Conclusiones. El conjunto de estas consideraciones permite extraer lecciones sobre desafíos y buenas prácticas, así como cuestiones a profundizar en el futuro. Contribuye, en este sentido, hacia una problematización creciente sobre las formas de producción, difusión y uso del conocimiento universitario en América Latina.
\end{abstract}

Palabras clave

Acceso abierto; Comunicación; Conocimiento; Repositorio digital; Universidad.

\section{Implementation of an open access strategy at the university: The case of UNVM (Argentina)}

\begin{abstract}
Objetive. The article addresses the experience of implementing an Open Access strategy at the National University of Villa María, Argentina. This initiative is part of Law 26,899 "Creation of Digital Institutional Repositories of Open Access, Own or Shared" (2013), which establishes the availability of all scientific production financed with public funds.

Methodology. Based on own participation as members of the Program: Production and Circulation of Knowledge at the Public University (PyCCUP), the paper is presented as a case analysis based on a systematization of experiences.

Results. It detail four stages involved in the process: 1. diagnosis, 2. awareness, training and conciliation of interests 3. institutional adjustment and, 4. technical and design aspects.

Conclusions. These considerations draw lessons on challenges and good practices, as well as issues to deepen in the future. It contributes, in this sense, towards a growing problematization on the ways of production, dissemination and use of university knowledge in Latin America.
\end{abstract}

Keywords

Communication; Digital repository; Knowledge; Open access; University. 


\section{Introducción}

Hace más de una década que universidades y organismos de CyT de la región debaten sobre los desafíos de las nuevas tecnologías de la información y la comunicación (TIC) para la producción, gestión y circulación del conocimiento. Una de estas posibilidades es la implementación de modelos de Acceso Abierto (AA). Los mismos implican que los usuarios de la producción científico-tecnológica pueden, en forma gratuita, leer, descargar, copiar, distribuir, imprimir, buscar o enlazar textos completos de publicaciones científicas, y usarlas con propósitos legítimos ligados a la investigación, el desarrollo tecnológico, la innovación, educación o gestión de políticas públicas, sin otras barreras económicas, legales o técnicas que las que suponga la Internet en sí misma.

En el caso de Argentina, un avance en esta dirección fue en 2013 la Ley 26.899 de Creación de Repositorios Digitales Institucionales de Acceso Abierto, Propios o Compartidos, la cual establece la puesta en disponibilidad de la producción científica financiada con fondos públicos, a través de la creación del Sistema Nacional de Repositorios Digitales. En tal contexto, la Universidad Nacional de Villa María (UNVM) conforma en 2014 el Programa: Producción y Circulación del Conocimiento en la Universidad Pública (PyCCUP), iniciativa que busca articular acciones en el marco de una estrategia integral de AA.

El artículo comienza por contextualizar la problemática y algunas de sus implicancias, a partir de declaraciones, definiciones, rutas de acceso y licenciamiento. Entendemos que el AA supone una serie de beneficios respecto a modelos tradicionales de divulgación científica, con mayores potencialidades en los países latinoamericanos. El AA contribuye, por otra parte, a una efectivización de derechos a la educación y la cultura, volviendo a problematizar la función social de las universidades, en especial aquellas de carácter público.

Basados en la propia participación como miembros del PyCCUP, el artículo presenta un estudio de caso en base a la sistematización de la experiencia en curso, la cual implicó instancias de diagnóstico, conciliación de intereses y adecuaciones institucionales. Entre ellas se realizaron tareas de sensibilización y capacitación a la comunidad universitaria, actualización de reglamentaciones vigentes, revisión de aspectos legales y licencias, aspectos técnicos y de diseño, entre otras.

En su conjunto, la estrategia involucró un debate sobre políticas de AA y su congruencia con las prioridades, recursos y necesidades de la institución, problematizando el cómo y para qué del conocimiento que se produce en esa casa de estudios.

\section{Universidades, conocimiento y sociedad}

Desde los albores de su existencia, las universidades fueron instituciones sociales centradas en la concentración y circulación del conocimiento. La legitimidad de esa función social asociada con la distribución del saber se basó en una serie de ideas sobre un conocimiento universal y elitista (Kuklinski, 2010).

Aunque los debates sobre la función social de la universidad exceden la intención de este escrito, podemos señalar que el acceso a la producción científica es una problemática clave que ha ganado relevancia en los últimos tiempos. Ya sea el acceso "desde fuera", al conocimiento producido en la universidad por cualquier individuo interesado en él; como el acceso "desde dentro", que requieren para el cumplimiento de sus labores los miembros de la comunidad universitaria: estudiantes, docentes e investigadores. En ambos casos, el acceso sin restricciones se ha tornado una demanda sobre la cual la institución universitaria busca dar respuestas.

En cuanto al acceso "desde fuera", resulta coherente que las instituciones financiadas por el Estado coloquen a disposición su producción científica sin restricciones económicas o de otro tipo. En caso contrario, se produce una dinámica comprendida como el modelo cerrado de investigación y divulgación universitaria: las universidades financian las investigaciones, los investigadores publican en revistas científicas de editoriales prestigiosas bajo estricto copyright y con suscripciones onerosas que deben ser pagadas nuevamente por las bibliotecas universitarias, para que su comunidad tenga acceso al conocimiento originalmente producido en la misma universidad.

Se termina de tal modo produciendo una privatización del conocimiento financiado con fondos públicos, más allá de lo cual el modelo alcanza una baja performance en términos de difusión e impacto social (Sempere García, 2013). Restringir el acceso a información generada con financiamiento público para alimentar modelos de negocio monopólicos, ajenos a la propia universidad, no resiste un análisis en el cual se considere tal función social como productora de nuevo conocimiento.

En cuanto al acceso "desde dentro", la situación actual reviste contradicciones entre el marco legal vigente y las prácticas cotidianas de estudiantes, docentes e investigadores. Las actividades de traducción, adaptación, digitalización o compilación de fragmentos están expresamente prohibidas por legislaciones como la de Argentina 
-en función de la Ley 11.723 y sus modificatorias ${ }^{1}$ - a excepción de autorización expresa del titular de los derechos. El derecho de cita permite el uso de hasta 1000 palabras de una obra textual o 7 compases de una obra musical.

Las excepciones educativas contempladas en otros regímenes legales, tales como el "uso justo" (presente en la legislación de EEUU) para el cual suelen calificar los usos educativos, o el derecho de copia privada, no existen en el caso argentino (Monroy Rodríguez, 2009). Sumado a otro conjunto de prohibiciones, terminan ubicando a esta legislación entre las más restrictivas del mundo².

A pesar de este carácter, la escasa vigilancia de su cumplimento genera desconocimiento y falta de interés entre los principales afectados. Las infracciones al derecho de autor son así una práctica instalada en la comunidad universitaria argentina, que rara vez implica las sanciones penales que la legislación prevé ${ }^{3}$. A esto se suma el acceso y utilización generalizada de materiales digitales, sin hacer distinción entre sus condiciones de uso.

El AA surge así en un contexto signado por la creciente tensión entre las regulaciones en materia de propiedad intelectual y el ejercicio de ciertos derechos humanos fundamentales. El derecho a la información, la educación, el acceso y participación en la vida cultural ha sido refrendado en la mayoría de los países a partir de pactos internacionales que definen sus alcances. A partir de allí, comienza a visibilizarse la necesidad de garantizar un equilibrio entre el reaseguro de la propiedad intelectual y el ejercicio efectivo de tales derechos, lo cual implica, en definitiva, una adecuación de los marcos legislativos vigentes (Chapman, 2012; Busaniche, 2016).

Estudios recientes remarcan aquí, más allá de sus consideraciones "técnicas", el sentido político del AA. Este sería capaz de subvertir ciertas prácticas perjudiciales para la ciencia latinoamericana derivadas de modelos tradicionales de publicación científica. Las presiones de los sistemas de CyT por alcanzar una alta calidad y visibilidad, terminan forzando a los investigadores a colocar sus resultados de investigación en revistas extranjeras privadas, dominadas por el idioma inglés y los académicos de un conjunto de universidades del norte junto a una agenda de temas de investigación no necesariamente coincidentes con los problemas locales. Más allá de esto, la producción de la región aparece subrepresentada y subestimada dentro de los circuitos dominantes de difusión del conocimiento (Aguado-López y Vargas Arbeláez, 2016).

Resulta además convergente con otras iniciativas como la Ciencia Abierta y los Datos Abiertos, nuevos enfoques sobre los procesos de producción y uso de conocimiento basados en el trabajo colaborativo a través de tecnologías digitales (RIN/NESTA, 2010).

\section{Acceso Abierto al conocimiento científico}

El movimiento de Acceso Abierto, con existencia desde 2002, se inscribe en la tradición inaugurada por el movimiento de software libre y su ampliación en la cultura libre: un conjunto heterogéneo de iniciativas que promueven la premisa de compartir el conocimiento y sus formalizaciones en bienes intangibles, sin restricciones de ningún tipo (Lessig, 2004; Busaniche, 2010). Por contraposición a la filosofía del copyright, el movimiento de AA sostiene que el conocimiento es un bien común, no rival, que se beneficia de la amplia circulación.

El AA se enmarca en un conjunto de iniciativas vinculadas a potenciar la circulación de la producción científica y académica. Si bien estas ideas tienen relación con dinámicas propias del campo científico ${ }^{4}$, la difusión de las TIC en las últimas décadas ha generado un entorno técnico ideal para la circulación de conocimiento en formatos digitales. En este sentido, y de forma más abarcativa, las prácticas de descarga, visualización, remezcla e interacción en línea entre millones de personas, constituyen el marco de una cultura de la conectividad actual (Van Dijck, 2013).

Las declaraciones del movimiento de AA se realizaron subsecuentemente en Budapest (2002), Bethesda (2003) y Berlín (2003). Otras iniciativas como la Open definition han buscado sistematizar tanto la definición como las licencias que se adecuan a los términos del AA, en paralelo a lo que hiciera el proyecto Freedom defined en 2005 (Freedom defined, 2016).

La Open definition explicita de modo genérico los permisos que una licencia de AA debe obligatoriamente dar a los receptores de una obra: uso libre, posibilidad de modificar, usar partes de la obra, compilar con otras obras en una nueva, permitir el acceso a una versión editable o en un formato abierto que no imponga el uso de un lector

\footnotetext{
${ }^{1}$ Ley 11.723 - Régimen Legal de la Propiedad Intelectual, cuyo articulado original data de 1933.

${ }^{2}$ Al respecto véase el Informe del proyecto IP Watch de Consumers International (2009a).

${ }^{3}$ Una excepción notable que movilizó a una parte significativa de la comunidad universitaria ya sensibilizada respecto de estos temas, fue el caso del profesor Horacio Potel (UNLanus) en 2009. Potel, creador de los sitios web www.heideggeriana.com.ar y www.jacquesderrida.com.ar fue demandado por violación a los derechos de autor de los autores mencionados (IP Watch, 2009b).

${ }^{4}$ Podemos mencionar aquí el ethos comunista de la ciencia enunciado por Merton, que define al conocimiento como propiedad colectiva, producto de la colaboración social, donde se aprecian la honestidad intelectual y la originalidad (Merton, 1977).
} 
no libre, y no imponer costos de ninguna índole como condición de acceso. Peter Suber, uno de los principales impulsores del AA, lo define estableciendo diferencias específicas con otros tipos de producción intelectual:

La literatura de acceso abierto (Open-access, OA) es digital, en línea, sin costo y sin la mayoría de las restricciones de derechos de autor y licencias. Lo que lo hace posible es Internet y el consentimiento del autor o titular de los derechos de autor.

En la mayoría de los campos, las revistas académicas no pagan a los autores, que por lo tanto pueden acceder a OA sin perder ingresos. En este sentido, los académicos y científicos se encuentran situados de forma muy diferente a la mayoría de los músicos y cineastas, y las controversias sobre OA con la música y el cine no se trasladan a la literatura de investigación. (...)

La literatura de OA no se produce de manera gratuita, aunque es menos costosa que la aquella publicada convencionalmente. La pregunta no es si la literatura académica se puede hacer sin costo, sino si hay mejores formas de pagar las cuentas que cobrando a los lectores y creando barreras de acceso. Los modelos de negocio para pagar las cuentas dependen de cómo se entrega el OA. (Suber, 2004 [traducción propia])

El movimiento de AA entiende que el tipo de información que las universidades producen y procesan se diferencia de otros tipos de conocimiento disponibles en Internet, por la calidad derivada del proceso de revisión por pares. El AA puede a su vez dividirse en dos rutas: AA oro, a través de revistas, y AA verde, por medio de repositorios. Las revistas científicas someten las contribuciones a evaluación por pares, para determinar su pertinencia y calidad. Los repositorios, por su parte, son bases de datos y gestores de contenidos online. Estos no realizan su propia revisión por pares, pero pueden albergar artículos revisados publicados en otros medios. Además, es frecuente que alberguen versiones pre-print, tesis y disertaciones, libros o capítulos de libros, bases de datos y obras digitalizadas de la biblioteca de la propia institución (Suber, 2015: 261).

En el caso argentino, la Ley 26.899: Creación de Repositorios Digitales Institucionales de Acceso Abierto, Propios o Compartidos, sienta las bases para una política AA en organismos nacionales de CyT. La misma establece que:

\begin{abstract}
..deberán desarrollar repositorios digitales institucionales de acceso abierto, propios o compartidos, en los que se depositará la producción científico-tecnológica resultante del trabajo, formación y/o proyectos, financiados total o parcialmente con fondos públicos, de sus investigadores, tecnólogos, docentes, becarios de posdoctorado y estudiantes de maestría y doctorado. Esta producción científico-tecnológica abarcará al conjunto de documentos (artículos de revistas, trabajos técnico-científicos, tesis académicas, entre otros), que sean resultado de la realización de actividades de investigación. (Artículo 1)
\end{abstract}

Dicha Ley se refiere además a los datos primarios de investigación, los cuales deberán estar disponibles en un plazo no mayor a cinco años del momento de su recolección (Artículo 5).

En la región, Argentina, Perú y México cuentan ya con leyes de AA, algunas todavía en proceso de reglamentación ${ }^{5}$ (Rogel Salazar, 2015). A nivel global, la Estrategia de AA de la UNESCO, fue aprobada en la Sesión 187a del Comité Ejecutivo y adoptada por unanimidad por la 36a Conferencia General en noviembre de 2011 (Packer et al., 2014).

Aunque se trata de un conjunto de iniciativas recientes, ya se están produciendo cambios e innovaciones institucionales fundamentales. En ese sentido, los primeros en dar cuenta de estas transformaciones e introducir la problemática del AA en las universidades han sido los bibliotecarios y profesionales de la información.

\title{
4 Estrategia de Acceso Abierto en la UNVM
}

El Programa: Producción y Circulación del Conocimiento en la Universidad Pública (PyCCUP) fue creado en 2014 nucleando a docentes, investigadores, equipos técnicos y de gestión de la Universidad. Se trató del primer programa avalado en conjunto por los tres Institutos que componen la UNVM: Ciencias Básicas y Aplicadas, Ciencias Sociales y Ciencias Humanas. (Res. IAPCByA 055/2014; IAPCS 202/2014; IAPCH 121/2014). Los objetivos del Programa se definieron del modo siguiente:

En vista de los procesos de transformación de las prácticas para buscar, acceder y generar nuevo conocimiento que desarrollan estudiantes, docentes e investigadores -especialmente en entornos digitalesconsideramos que la Universidad Nacional de Villa María debe acompañar críticamente tales transformaciones. Es menester para ello identificar y analizar las dimensiones epistemológicas, pedagógicas, legales y éticas involucradas, para favorecer el debate fundado y propositivo, así como la exploración de alternativas viables que tendrán en las universidades su espacio privilegiado de discusión e implementación, en tanto centros históricos de producción y circulación de conocimientos.

El Programa Producción y Circulación del Conocimiento en la Universidad Pública (PyCCUP) se plantea de este modo como un espacio orientado hacia el diagnóstico, la sensibilización y la capacitación, con el 
El Programa inaugura así un marco de trabajo en vistas a la creación de una estrategia AA. El proyecto toma por base la creación del repositorio institucional y otras iniciativas conexas, en tanto, herramientas de acceso y visibilización del conocimiento producido en la Universidad.

Como resultados principales, durante el 2015 se alcanzó un subsidio para financiamiento en infraestructura y se realizó el trabajo inicial de puesta en marcha del repositorio, lo cual incluyó tareas de testeo. Durante 2016 se comenzó la carga de elementos al repositorio, encontrándose ya disponible un lote inicial de contenidos.

Hasta el momento se crearon además dos revistas académicas con revisión por pares, ambas bajo la plataforma Open Journal System (OJS). La primera, Raigal. Revista Interdisciplinaria de Ciencias Sociales, ofrece una publicación para investigaciones recientes, sometida a revisión por pares y con altos estándares en términos de calidad y visibilidad. La segunda, Sociales Investiga. Escritos Académicos de Extensión y Docencia, apunta al intercambio con el medio y la comunidad local, ofreciendo secciones flexibles para la publicación de comunicaciones y resultados de investigación. El trabajo editorial de las revistas implica la consolidación de un cuerpo de evaluadores, el cumplimiento de estándares y el posicionamiento en buscadores e índices regionales.

Hacia el futuro se presenta el desafío de incorporar otro de los elementos previstos por la Ley 26.899: el acceso a datos primarios de investigación. Tal iniciativa está ya suscitando debates en cuanto a sus alcances y posibilidades.

A continuación detallamos cuatro momentos involucradas en la creación de la estrategia AA en la universidad: 1. diagnóstico, 2. sensibilización, capacitación y conciliación de intereses, 3. adecuación institucional y 4. aspectos técnicos y de diseño. El conjunto de estas consideraciones sintetizan la experiencia llevada adelante hasta el momento, y permiten realizar consideraciones sobre lecciones aprendidas y cuestiones a profundizar hacia el futuro.

\subsection{Diagnóstico}

Como parte del diagnóstico se sondearon procesos en marcha en otros centros y se elaboró un listado de portales de referencia, los cuales fueron analizados por la comisión. Existen valiosas experiencias en el país y el exterior, que permiten anticipar problemas e implementar mejores prácticas. En el caso argentino, encontramos el repositorio de la Universidad Nacional de La Plata (SEDICI Sitio web, 2016) y la Universidad Nacional de Córdoba, que cuenta con una Oficina de Conocimiento Abierto (OCA Sitio web, 2016). En cuanto a la región de habla hispana, destacan los casos de la Universidad de Salamanca, Universidad Autónoma de Nuevo León y la Universidad Autónoma del Estado de México, entre otras.

En el contexto global de acceso a volúmenes inéditos de información, la posibilidad de concentrar la producción local y tornarla accesible a través de un repositorio institucional constituye una oportunidad especialmente para una universidad joven y con perfil de desarrollo regional como la UNVM. Ella cuenta con una cantidad de proyectos de investigación concluidos y en curso, además de producción docente, trabajos finales de grado y posgrado. También cuenta con materiales educativos, documentales y de extensión, así como producciones multimediales de diversos formatos. El desafío inicial consistió entonces en ponderar cuáles de estos materiales estaban en condiciones de ser colocados en el repositorio y cómo se llevaría a cabo tal tarea.

Identificamos además una falta de familiaridad con aspectos relacionados a la propiedad autoral, tema en el que se evidenció un conocimiento acotado o impreciso. Algunos aspectos destacados son:

- incomprensión del marco legal regulatorio vigente de las obras autorales en Argentina, tanto desde el rol de autores, como desde el rol de consumidores y "prescriptores" de obras autorales a los estudiantes.

- desbalance entre la valoración simbólica de los derechos de autor de la propia producción, incluso más allá de las cesiones firmadas con editoriales; en contraste con el desinterés o desconocimiento por el respeto de los derechos de autor de las obras de otros creadores, especialmente aquellas en formato digital.

- incompatibilidad entre las exigencias o sugerencias de los sistemas de evaluación de la producción científica y académica, que suponen la publicación en revistas de alto impacto pertenecientes al modelo cerrado, no afines al AA.

En cada uno de los tres Institutos de la Universidad se presentaron, a su vez, problemáticas particulares. En el caso de Ciencias Básicas y Aplicadas, existe interés por implementar una política de patentamiento que genere ingresos adicionales para futuras investigaciones. En el caso de Ciencias Sociales, se observó la necesidad de 
establecer una política para prevenir y controlar el plagio. En el caso de Ciencias Humanas existen complejidades adicionales, dado que las producciones artísticas o multimediales y el frecuente remix de contenidos requieren un tratamiento especial.

Se ponderó además cuáles serían las áreas involucradas en la creación y gestión del repositorio. Se estableció que su gestión quedaría en manos de la Biblioteca Central, la cual solicitó una ampliación de su personal para tal fin. La Dirección de Informática evaluaría los recursos de infraestructura necesarios y pondría en marcha la plataforma. Por su parte, las autoridades de cada Instituto se comprometieron a ordenar las reglamentaciones vigentes.

\subsection{Sensibilización, capacitación, conciliación de intereses}

Una de las acciones iniciales consistió en una campaña de sensibilización y capacitación. Durante 2014 se realizaron talleres sobre AA, los cuales contaron con alrededor de setenta docentes, investigadores y estudiantes de posgrado. Se pretendió con ello introducir la problemática y visibilizar en qué medida resultaba relevante para la producción académica local, junto a sus implicancias técnicas y políticas. Se realizaron además sucesivos encuentros con especialistas para intercambiar y sacar provecho de experiencias exitosas de implementación.

En los encuentros con actores universitarios se detectaron problemáticas similares a las planteadas por otros centros: cierta sobrevaloración de los beneficios que supone para la circulación de las producciones científicas y académicas la retención de "todos los derechos", confusión respecto de la cesión de algunos derechos con la pérdida de los derechos morales (el derecho a ser reconocido siempre como el autor de una obra), y desconfianza respecto de los efectos perjudiciales que podrían generar las obras derivadas. Es importante recordar que las obras derivadas incluyen traducciones y adaptaciones con fines didácticos, o compilaciones de varios fragmentos de obras en una nueva, sólo por citar prácticas frecuentes en la tarea docente.

Tales actividades convergieron en la realización de la Semana del Acceso Abierto, una iniciativa que se celebra a nivel global. A fines del 2015 y durante las Jornadas Anuales de Investigación se realizó un primer Panel con expertos. En 2016 se sumaron conferencias sobre propiedad intelectual, presentación de libros y talleres de cultura libre, abordando temas como la edición colaborativa en Wikipedia, aplicaciones de software y hardware libre, así como recursos educativos abiertos.

También se inició en 2015 el Espacio Curricular Optativo para estudiantes de Ciencias Sociales "Apropiación social de la tecnología y nuevas formas de comunicación”. En 2016, se formó asimismo el Proyecto de Investigación: Paradigmas tecnológicos libres, recursos educativos abiertos y apropiación de la tecnología. Prácticas de aprendizaje de jóvenes escolarizados en Córdoba (PIC- UNVM 2016-17).

Como parte del acercamiento a los actores implicados en la creación y sostenimiento de un repositorio institucional, se convocó a la editorial universitaria local (EDUVIM) para discutir posibles formas de articulación. Una problemática aquí son los contratos de cesión exclusiva de derechos, que la mayoría de las editoriales universitarias firman con los autores. Esta constituye una tradición de edición vinculada al soporte papel -con sus costos de impresión, logística y difusión asociados- que puede resultar antagónica al modelo AA. Al respecto es importante recordar que las licencias libres permiten usos comerciales de los materiales publicados, siempre que no sean de tipo exclusivo. Es decir, si una obra se encuentra publicada bajo una licencia Creative Commons Atribución (licencia recomendada para el AA), la obra puede ser comercializada y/o modificada por cualquiera que agregue valor y logre crear un nuevo modelo de negocio respecto de la publicación original.

Otro actor relevante es la Oficina de Propiedad Intelectual y Transferencia Científico Tecnológica, la cual busca implementar una política de patentamiento en relación con desarrollos industriales, principalmente para el sector del agro y alimentos. La reglamentación de la Ley 26.899 , en sus definiciones generales, especifica que el AA no debería entenderse en colisión con la propiedad intelectual y el sistema de patentes de invención (Resolución 753E/2016). Es necesario, en este sentido, avanzar en una política integral para el cuidado y la promoción de la producción de la universidad, que fomente la innovación y la apropiación social del conocimiento.

\subsection{Adecuación institucional}

Los contenidos de los repositorios de AA son en su mayoría obras autorales, reguladas por derechos de autor. En el desarrollo de esa función, la gestión de los derechos de autor de las obras a incorporar al repositorio, suponen, un aspecto crucial para el crecimiento de su acervo.

La creación de un repositorio institucional genera un parteaguas entre la producción generada con anterioridad, la cual no tuvo en cuenta las condiciones del AA, y las futuras producciones, sobre las cuales se deben tomar recaudos para facilitar su incorporación. En el primer caso, es necesario un trabajo de selección y adecuación del material, lo cual supone un proceso lento y costoso. En el segundo caso, se deberán realizar actualizaciones sobre las reglamentaciones vigentes de modo de asegurar pautas de presentación así como la elección de una licencia compatible con el repositorio. 
En función de la experiencia de instituciones de referencia, se identificaron y analizaron las reglamentaciones que eran necesario adaptar. Entre ellas podemos mencionar:

- reglamento de los trabajos finales de grado y tesis de posgrado: exige copias impresas y en soporte digital obsoleto, sin cesión de derechos para la publicación digital. Se encuentra en desarrollo una propuesta de reglamento que elimine la versión impresa y automatice la cesión de derechos de la versión digital con una licencia de AA. La propuesta debe considerar situaciones especiales, como las producciones que incluyan materiales de otros autores (imágenes, video, audio) y que requieran cesión específica y compatible, o una versión editada que no incluya esos materiales, a los fines de su carga en el repositorio institucional.

- reglamento sobre informes y resultados de investigación financiados por la universidad: las propuestas discutidas se encontraron en cierta contradicción entre las exigencias de los evaluadores de organismos externos, respecto de las publicaciones recomendadas y formas de circulación que éstas sostienen. Esta problemática excede el ámbito de acción de la universidad y señala uno de los desafíos inmediatos que la reglamentación de la Ley 26.899 debe contemplar. Más allá de estos casos, la restante producción científica en investigación puede sumarse al repositorio institucional con una adecuación de la reglamentación vigente.

- resúmenes y ponencias en actas de eventos científicos organizados por la universidad: Una reglamentación general podría solicitar que toda convocatoria a presentación de ponencias implique la cesión de los derechos bajo una licencia de $\mathrm{AA}$, a los fines de la inclusión en el repositorio institucional, ya sea para su publicación en actas o como artículos individuales. Esta iniciativa confluye con la implementación de plataformas para la gestión de eventos académicos, como es el caso de Open Conference System (OCS).

En cuanto a la implementación de licencias, encontramos un escenario confuso en algunos de los repositorios analizados: desde el uso de variedad de licencias, no necesariamente incluidas en los materiales descargados, lo cual dificulta su identificación fuera de contexto; hasta uso de licencias en contradicción con la definición de AA (caso de la Creative Commons Sin usos comerciales y Sin Obras derivada), que no permiten traducciones, adaptaciones ni compilaciones. De acuerdo con la definición de AA, se decidió finalmente optar por la licencia más permisiva dentro del sistema Creative Commons: Atribución 4.0 Internacional.

\subsection{Aspectos técnicos y de diseño}

La introducción de un sistema de gestión como el repositorio, requiere de personal dedicado específicamente a esa tarea capacitado para llevarla a buen fin, así como de infraestructura de almacenamiento y transferencia de datos para que los contenidos se encuentren a disposición siempre que sean requeridos.

La creación del repositorio institucional requirió, por ello, la consideración de aspectos técnicos referidos a la infraestructura tecnológica, la elección del software para la plataforma, así como formatos y diseño de los objetos digitales a incluir. En función de ello, la comisión contó desde el inicio con un representante del área de informática de la universidad. Fue fundamental a lo largo del proceso establecer un diálogo entre las posibilidades técnicas, las decisiones institucionales que la estrategia AA implicaba.

En relación al software a utilizar, el Sistema Nacional de Repositorios Digitales (SNRD) recomienda una serie de alternativas que garantizan confiabilidad e interoperabilidad. Los sondeos realizados por la comisión resultaron favorables a la instalación del sistema DSpace, el más utilizado en universidades argentinas. En función de que el área de biblioteca contaba con experiencia previa con PMB, un software francés de código abierto utilizado en 500 bibliotecas y centros de documentación a nivel global, se optó finalmente por esta herramienta de gestión de colecciones digitales.

Respecto de los aspectos de diseño y edición de los objetos digitales, nuestro análisis de repositorios institucionales de referencia concluyó que se requiere una labor de curado, para garantizar que las cargas se completen adecuadamente y no se realicen infracciones a los derechos de autor. También una tarea de edición básica, que resguarde la identidad visual institucional. En función de ello, no será posible la opción de autoarchivo, esto es, la carga por parte de los propios autores. Los contenidos serán procesados por el área de biblioteca previo a su puesta en disponibilidad. 


\section{Conclusiones}

A lo largo del escrito se describió el proceso y algunas de las decisiones implicadas en la implementación de una estrategia de AA en la UNVM. En un nivel más amplio, se presentó la iniciativa en el contexto de los debates e iniciativas contemporáneas en torno a la circulación del conocimiento, así como los alcances de la Ley 26.899 en Argentina. El AA supone la posibilidad de acceder a conocimientos e investigación actualizada y de alta calidad, tanto los que se producen de manera local como en otros centros científicos del mundo.

Como política de difusión de la producción científica y académica, el AA supone varios niveles de aproximación. En primera instancia, se enfoca en el tema central de la función social de la universidad, en especial las de carácter público. A nivel institucional, es una estrategia de visibilización de la propia producción, tanto más relevante cuanto más localizada y específica sea dicha producción y más limitadas sus formas de circulación previas. En el mismo sentido, representa una oportunidad de interacción y cooperación a nivel nacional y global.

Una estrategia de AA implica consideraciones tanto técnicas, normativas, legales y logísticas. A partir de la experiencia presentada, podemos extraer de manera sucinta las siguientes lecciones aprendidas:

- Se requieren acciones de sensibilización y capacitación recurrentes, que expliciten los alcances del AA. Existe, en general, un desconocimiento respecto de los circuitos de publicación y las condiciones uso de la producción científica.

- La adecuación al AA requiere decisiones consensuadas y una convicción de los actores involucrados, para materializarse luego en acciones concretas que favorezcan su implementación.

- La adaptación al AA implica actualizaciones en prácticas institucionales, académicas y científicas, las cuales, una vez establecidas, allanan el camino. En este proceso pueden imitarse buenas prácticas ya empleadas por centros pioneros.

- La implementación de una estrategia AA requiere de infraestructura y personal especialmente calificado. Se debe procurar no sobrecargar los recursos ya existentes.

- El rol de las bibliotecas resulta central, no sólo como administradoras del repositorio, sino como sostenedoras de una cultura del AA.

Nuestra participación buscó compartir avances de un proceso en curso, junto con los debates y adecuaciones institucionales suscitadas. Intentamos con ello aportar hacia una problematización creciente en torno a las formas de producción, difusión y utilización del conocimiento universitario en el contexto de América Latina.

\section{Referencias}

Aguado-López, E., \& Vargas Arbeláez, E. (2016). Reapropiación del conocimiento y descolonización: el acceso abierto como proceso de acción política del sur. Revista Colombiana de Sociología, 39(2), 69-88.

Busaniche, B. (Ed.). (2010). Argentina copyleft. Córdoba: Vía Libre.

Busaniche, B. (2016). Propiedad intelectual y derechos humanos. Hacia un sistema de derechos de autor que promueva los derechos culturales. Temperley: Tren en Movimiento.

Chapman, A. R. (2001). La propiedad intelectual como derecho humano (obligaciones dimanantes del apartado c) del párrafo 1 del Artículo 15 del Pacto Internacional de Derechos Económicos, Sociales y Culturales). Boletín de derecho de autor, XXXV(3), 4-39. Recuperado el 10 de julio de 2016 de http://unesdoc.unesco.org/images/0012/001255/125505s.pdf 
IP Watch (2009a). IP Watch de Consumers International 2009. Recuperado el 10 de julio de 2016 de https://www.opensocietyfoundations.org/sites/default/files/ip-watch-list-20100220.pdf

Lessig, L. (2004). Cultura libre. Madrid: Traficantes de Sueños.

IP Watch (2009b). Un caso sobre derechos de autor en Argentina pone en primer plano el acceso a la educación. Recuperado el 10 de julio de 2016 de http://www.ip-watch.org/2009/05/18/un-caso-sobre-derechos-de-autor-en-argentina-pone-en-primerplano-el-acceso-a-la-educacion/

Ley 11.723: Régimen Legal de la Propiedad Intelectual. Boletín Oficial de la República Argentina, Buenos Aires, 26 de Septiembre de 1933. Recuperado el 10 de julio de 2016 de http://servicios.infoleg.gob.ar/infoleglnternet/anexos/4000044999/42755/texact.htm

Ley 26.899: Creación de Repositorios Digitales Institucionales de Acceso Abierto, Propios o Compartidos. Boletín Oficial de la República Argentina, Buenos Aires, 13 de noviembre de 2013. Recuperado el 10 de julio de 2016 de http://repositorios.mincyt.gob.ar/recursos.php

Merton, R. K. (1977) [1973]. La sociología de la ciencia: investigaciones teóricas y empíricas. Recopilación e introducción de Norman W. Storer. Madrid: Alianza Editorial.

Monroy Rodríguez, J.C. (2009). Estudio sobre las limitaciones o excepciones al derecho de autor y los derechos conexos en beneficio de las actividades educativas y de investigación en América latina y el Caribe. Comité permanente de derecho de autor y derechos conexos. Decimonovena sesión Ginebra, OMPI. Recuperado el 10 de julio de 2016 de http://www.wipo.int/edocs/mdocs/copyright/es/sccr 19/sccr 19 4.pdf

Packer, A. L., Cop, N., Luccisano, A., Ramalho, A., \& Spinak, E. (2014). SciELO-15 años de acceso abierto: un estudio analítico sobre acceso abierto y comunicación científica. París: Unesco.

Raffo, J. (2010). Derecho autoral. Hacia un cambio de paradigma. Buenos Aires: Marcial Pons.

Resolución 753-E/2016, Ministerio de Ciencia, Tecnología e Innovación Productiva. Boletín Oficial República Argentina, Buenos Aires, 10 de noviembre de 2016

RIN/NESTA (2010). Open to All? Case studies of openness in research. London: Research Information Network and Nationa Endowment for Science, Technology and the Arts. Recuperado el 10 de julio de 2016 de http://www.rin.ac.uk/system/files/attachments/NESTA-RIN Open Science V01 0.pdf

Rogel Salazar, R. (2015). Acceso Abierto, información científica disponible en línea sin barreras. Revista Digital Universitaria 16(3). Recuperado el 10 de julio de 2016 de http:/www.revista.unam.mx/vol.16/num3/art19/index.html

Sempere García, T. (2013). Public Access to Publicly Funded Materials: What Could Be. Entrada de blog. Recuperado el 10 de julio de 2016 de https://creativecommons.org/2013/09/25/public-access-to-publicly-funded-materials-what-could-be/ 
Suber, P. (2004). A Very Brief Introduction to Open Access. Recuperado el 10 de julio de 2016 de http://legacy.earlham.edu/ peters/fos/brief.htm

Suber, P. (2015). Acceso abierto. Traducción de Remedios Melero. Toluca: Universidad Autónoma del Estado de México.

Van Dijck, J. (2013). The culture of connectivity: A critical history of social media. New York: Oxford University Press.

\section{Sitios web}

Freedom Defined. Definición de las obras culturales libres. Recuperado el 10 de julio de 2016 de http://freedomdefined.org/Definition/Es

Oficina de Conocimiento Abierto (OCA), Universidad Nacional de Córdoba. Recuperado el 10 de julio de 2016 de http://www.artec.unc.edu.ar/oca

Open definition. Conformant Licenses. Recuperado el 10 de julio de 2016 de http://opendefinition.org/licenses/

Repositorio Digital, Biblioteca Central UNVM. Recuperado el 10 de julio de 2016 de http://biblioteca.unvm.edu.ar/Repositorio/index.html

SEDICI - Repositorio de la Universidad Nacional de La Plata. Recuperado el 10 de julio de 2016 de http://sedici.unlp.edu.ar/ 


\section{Datos de los autores}

Mauricio Alejandro Grasso

Esp. en Investigación de la Comunicación. Docente en la Facultad de Ciencias de la Comunicación de la Universidad Nacional de Córdoba y el Instituto Académico Pedagógico Ciencias Sociales de la Universidad Nacional de Villa María, Argentina.

mgrasso@unvm.edu.ar

\section{Lila Isabel Pagola}

Esp. en Tecnologías Multimedia para Desarrollos Educativos. Docente en la Facultad de Arte y Diseño de la Universidad Provincial de Córdoba y los Institutos Académico Pedagógico Ciencias Sociales y Ciencias Humanas de la Universidad Nacional de Villa María.

Ipagola@unvm.edu.ar

Agustín Zanotti

Dr. en Estudios Sociales de América Latina. Investigador en la Facultad de Ciencias Sociales de la Universidad Nacional de Córdoba y el CONICET (Centro de Investigaciones y Estudios sobre Cultura y Sociedad). Docente del Instituto Académico Pedagógico Ciencias Sociales de la Universidad Nacional de Villa María, Argentina.

azanotti@unvm.edu.ar

Recibido - Received: 2017-02-02

Aceptado - Accepted: 2018-12-11

\section{$(\mathrm{cc}) \mathrm{BY}$}

This work is licensed under a Creative Commons Attribution 4.0 United States License.

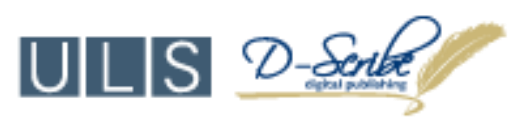

This journal is published by the University Library System of the University of Pittsburgh as part of its D-Scribe Digital Publishing Program and is cosponsored by the University of Pittsburgh Press. 